to form a twin transmission line roughly a quarter wave-Iength long, and surrounded, to eliminate radiation losses, by a cylindrical sheath, which was either silver-plated or lined with lead-foil to minimize resistive losses. Measurements of the width of the resonance curve of the system by a controlled variation of the oscillator frequency gave a value for the skin resistance of the specimen.

The accompanying graph shows the skin resistance $R$ of tin and mercury, relative to its value $R_{n}$ for the normal metal just above the mercury, relative to its value $R_{n}$ for the normal metal just above the
transition point, as a function of temperature. The shape of the curve for tin is in good agreement with London's published curve, measured for the slightly different frequency of 1,500 Mc./sec.; there are no previous results with which to compare the curve for mercury. For previous results with which to compare the curve for mercury. For is some uncertainty in the values of the skin resistance at the lowest is some uncertainty in the values of the skin resistance at the lowest of the resonator to about 250,000 . By comparing the results for two
o the of the resonator to about 250,000 . By comparing the results for two
wires of different diameter, the dielectric loss mav be estimated, wires of different diameter, the dielectric loss mav be estimated, as possible.

The absolute skin resistance of the specimen may be determined from the $Q$ and the geometry of the resonator, and from this, by from the $Q$ and the geometry of the resonator, and from this, by
application of the standard theory of the skin effect, the specific application of the standard theory of the skin effect, the specific
conductivity of the metal can be deduced. The value obtained for conductivity of the metal can be deduced. The value obtained for normal tin at $3.8^{\circ} \mathrm{K}$. was $2 \cdot 1 \pm 0.2 \times 10^{7} \mathrm{ohm}^{-1} \mathrm{~cm} .^{-1}$ (the large probable error arises from the approximations used in performing the calculation rather than from the measurements, which were consistent
within 1 per cent); a D.c. measurement of the specific conductivity within 1 per cent); a D.c. measurement of the specific conductivity
gave $15 \times 10^{7} \mathrm{ohm}^{-1} \mathrm{~cm}-{ }^{-1}$ more than seven times as great $\mathrm{A}$ disgave $15 \times 10^{7} \mathrm{ohm}^{-1} \mathrm{~cm} .^{-1}$ more than seven times as great. A dis-
crepancy of the same magnitude was noted by London. For mercury crepancy of the same magnitude was noted by London. For mercury
the effect is not so pronounced, the speciflc conductivity as deduced from the radio-frequency measurements being $0.92 \times 10^{7}$, compared with the D.C. value of $2.1 \times 10^{2}$

Although the shape of the specimen was not very suitable, an attempt was made to discover whether the skin resistance was affected by the presence of a constant magnetic field. Up to one half the critical field, no variation of skin resistance was detected, which by London's theory (see below) may be interpreted as evidence for the constancy of the penetration depth in small flelds. No useful readings could be taken in stronger flelds, since a portion of the specimen passed into the intermediate state.

The variation of the skin resistance of mercury with temperature is particularly interesting, since with the aid of London's ${ }^{1}$ theory of the effect it is possible to deduce the variation with temperature of the penetration depth $\lambda$, which has been studied for mercury more directly by other methods; it is, however, too much to expect agreement with these other results, since they do not agree among themselves. Appleyard and his collaborators ${ }^{3}$, from the properties of thin
films, and Shoenberg ${ }^{4}$, by measurements on colloids, found an increase in $\lambda$ as the critical temperature is approached from below, whereas

\title{
Investigations of Near Infra-Red Radiations by Means of Image Converters
}

For rapid examinations of near infra-red light up to $15,000 \mathrm{~A}$., image converters are very useful. Their main advantage lies in enabling one to 'see' immediately what infra-red sensitized plates show only after involved processing. The spectrum under test is projected The number of photo-electrons released is proportional to $\mathrm{Cs}, \mathrm{Ag}-\mathrm{Cs}$. The number of photo-electrons released is proportional to the intensity of radiation on that particular part of the cathode; they are focused by an electronic lens and form a picture on the fluorescent screen, which can either be studied visually or photographed. The accompanying illustration shows a photograph $(\times 1 \cdot 5)$ of the mercury spectrum. In addition to the visible lines a number of lines in the near infra-red part of the spectrum have appeared. On the original photographic be seen on the reproduction.

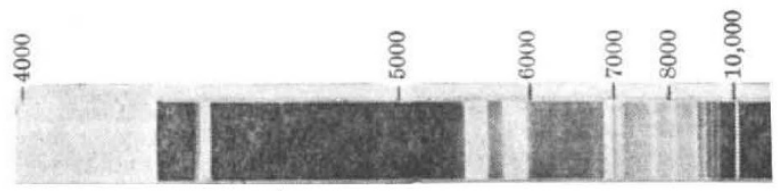

It should be mentioned that the converter used had a convex cathode, so that only a part of the image could be focused; but it appears probable that, with an improved apparatus of this type, a number of important problems could be attacked.

Physical Institute of Charles' University,

A. VASKO Praha II.

\section{Long Wave-length Absorption Bands of Aromatic Molecules}

CALCULATIONS of the long wave-length absorption bands of aromatic molecules, such as those carried out by Sklar ${ }^{1}$ and Förster ${ }^{2}$, give good agreement with the experimental values of absorption maxima even when only nearest-neighbour exchange effects are taken into account (as in Förster's work). In these calculations, it almost always happens that the lowest two totally symmetric levels (of which the ground-state is one) are separated by an unsymmetric level, and the transition to this from the ground-state is permitted in molecules having, at most, twofold axes of symmetry: experimentally. molecules have intensities compatible with an allowed transition-anthracene, for example, has $\varepsilon_{\max }$. of about $10^{4}$. Phenanthrene, however, provides an interesting departure from this usual situation. Here the energy-level calculation shows that there is no unsymmetric level between the groundstate and the lowest excited symmetric level, and consequently the transition $R / R_{n}$ of lowest energy is one between levels of the same symmetry. The usual symmetry considerations show that the transition moment for such a change will be very small and will lie along the axis of symmetry of the molecule. In fact, the absorption of phenanthrene does show a weak long wave-length absorption between $3000 \mathrm{~A}$. and 3600 A., having its centre at about $3400 \mathrm{~A}$., and with emax. about 300 . This band is comparable in intensity with the forbidden $A_{1 g}-B_{2} u$ transition in benzene $(2600$ A.) and is, apparently, to be associated with the symmetric-symmetric transition discussed above. Förster associated his calculated value of $3000 \mathrm{~A}$. with the strong phenanthrene band at 2950 A.; it now appears that it should be assigned to

Casimi ${ }^{5}$, using a mutual inductance method with macroscopic specimens, found no such change. In order to apply London's theory to the radio-frequency measurements, it is necessary to assume only one parameter $\lambda_{0}$, the penetration depth at $0^{\circ} \mathrm{K}$. Taking $\lambda_{0}=1.0 \times$ $10^{-5} \mathrm{~cm}$., as found by Appleyard et al., the value of $\lambda$ at any other temperature may be calculated to agree within 10 per cent with as very significant, in view of the assumptions involved in London's theory, which need to be tested by an extensive investigation of the behaviour of both normal metais and

Royal Society Mond Laboratory,

A. B. Pippard Cambridge.
July 22 .

${ }^{1}$ London, H., Proc. Roy. Soc., A, 176, 522 (1940). ' Appleyard, Bristow, London, H., and Misener, Proc. Roy. Soc., A, $172,540(1939)$

Shoenberg, Proc. Roy. Soe., A, 175, 49 (1940).

${ }^{5}$ Casimir, Leid. Comm., 261, c (1940).
$2950 \mathrm{~A}$. band is due to a symmetric-unsymmetric transition of higher energy. The poor agreement between the calculated and experimental values for the forbidden transition is connected with the fact that Förster's empirical exchange integral was estimated as an average for a number of inter-symmetry transitions and is too large for a symmetric-symmetric change.

The above assignment receives confirmation from the fact that the $3400 \mathrm{~A}$. band is greatly increased in intensity $(\times 10)$ when the $C_{8} 8$. syme Aand is greatly increased in inten carbon atom, to give the skew benzquinolines The situation for a carbon atom, to give the skew benzquinolines. The situation is in marked contrast to the anthracene-acridine-bena-g-quinoline case where the transition moment (this is an allowed transition) is scarcely
altered when the symmetry is lowered.

Further details, together with some related work, will be published soon.

Department of Chemistry

D. P. Craig

University of Sydney.

July 10.

1 Sklar, A. I., $J$, Chem, Phys, 5, 669 (1937),

2 Förster, Th., Z. Elektrochem., 45, 548 (1939). 